\title{
Letter
}

\section{Monitoring should not be a barrier to conservation success: a response to Sanders et al.}

Sanders et al. (2021) interviewed conservationists (mostly from Kenya and South Africa) to produce a useful typology of the main barriers to conservation success. They found that the inability to demonstrate impact was the second most commonly cited barrier of the 74 identified. Three main causes were proposed to explain this challenge (p. 250). Firstly, many interviewees felt they 'did not have the right tools to effectively assess conservation initiatives... because progress is not fast or linear and therefore impacts can be difficult to measure'. Secondly, 'reasons for success may not be obvious, making it difficult to define and report on them'. This led the authors to conclude that 'there is no satisfactory method for measuring impact consistently or attributing benefits to specific interventions'. I would argue that these are not causes of weak monitoring but oftrepeated excuses.

Several tools and approaches exist for defining and quantifying conservation success and for monitoring projects and their impacts on biodiversity. Available resources include management standards to define and design appropriate project goals and objectives around situation analyses that enhance the feasibility and measurability of conservation interventions (Conservation Measures Partnership, 2020). Such approaches include the use of scalable goals and linked indicator frameworks (sensu Stephenson, 2019) that allow the monitoring of responses, pressures, biodiversity state and benefits to people along a project's theory of change, facilitating the identification of actual and potential reasons for success. Impact evaluations (e.g. Gertler et al., 2016), including the use of randomized control trials (Pyengar et al., 2021) and before after control intervention analyses (Wauchope et al., 2021), can then be implemented to measure a project's success against counterfactuals that enhance attribution of results from project actions. As Sanders et al. note, progress is not always fast or linear, but that does not obviate the necessity and the usefulness of applying existing management best practices and evaluation techniques.

The top-rated barrier Sanders et al. identified-local capacity -is undoubtedly a factor behind weak monitoring and the poor adoption of existing tools, especially in Africa (O'Connell et al., 2019), and it is sometimes compounded by a lack of willingness to discuss and share results that may not always be favourable (Stephenson et al., 2017). The conservation community therefore needs to adopt a more results-based management culture and address capacity issues, especially in countries with rich biodiversity. Managers need to factor in a larger proportion of existing project resources, time and effort for data collection and use, and to build capacity for planning, monitoring and evaluation in their teams and partners. Donors need to ensure monitoring budgets are funded and become stricter at holding project managers accountable, while at the same time allowing the space to fail, learn and adapt. With a more widespread results-based management culture and the application of relevant monitoring and evaluation tools we will be able to quantify project progress and demonstrate the level of conservation impact. This will facilitate adaptive management, allowing successful project strategies to be replicated and less successful ones to be adapted. Monitoring should therefore be a solution, not a barrier, to conservation success.

P.J. STEPHENSON (๑ orcid.org/0000-0002-0087-466X) IUCN Species Survival Commission Species Monitoring Specialist Group, Laboratory for Conservation Biology, Department of Ecology \& Evolution, University of Lausanne, Biophore Building, CH-1015 Lausanne, Switzerland. E-mail stephensonpj@gmail.com

\section{References}

Conservation Measures Partnership (2020) Open Standards for the Practice of Conservation. Version 4. Conservation Measures Partnership, Bethesda, USA.

Gertler, P.J., Martinez, S., Premand, P., Rawlings, L.B. \& Vermeersch, C.M. (2016) Impact Evaluation in Practice. 2nd edition. The World Bank, Washington, DC, USA.

O’Connell, M., Nasirwa, O., Carter, M., Farmer, K., Appleton, M., Arinaitwe, J. et al. (2019) Capacity building for conservation: problems and potential solutions for sub-Saharan Africa. Oryx, 53, 273-283.

Pynegar, E.L., Gibbons, J.M., Asquith, N.M. \& Jones, J.P.G. (2021) What role should randomized control trials play in providing the evidence base for conservation? Oryx, 55, 235-244.

Sanders, M.J., Miller, L., Bhagwat, S.A. \& Rogers, A. (2021) Conservation conversations: a typology of barriers to conservation success. Oryx, 55, 245-254.

Stephenson, P.J. (2019) The Holy Grail of biodiversity conservation management: monitoring impact in projects and project portfolios. Perspectives in Ecology and Conservation, 17, 182-192.

Stephenson, P.J., Bowles-Newark, N., Regan, E., Stanwell-Smith, D., Diagana, M., Hoft, R. et al. (2017) Unblocking the flow of biodiversity data for decision-making in Africa. Biological Conservation, 213, 335-340.

Wauchope, H.S., Amano, T., Geldmann, J., Johnston, A., Simmons, B.I., Sutherland, W.J. \& Jones, J.P.G. (2021) Evaluating impact using time-series data. Trends in Ecology \& Evolution, 36, 193-205. 